\title{
Evaluación del índice de masa corporal y su relación con la hiperglucemia en pacientes diabéticos tipo 2, en Lima Metropolitana, durante el año 2011
}

Clever Arias, Rubén Valdivieso, Marco Núñez, Miriam Inga, Felipe Sosa, Oscar Acosta

Departamento Académico de Ciencias Dinámicas, Facultad de Medicina, UNMSM

Objetivos: Establecer una correlación entre IMC e hiperglicemia entre pacientes con diabetes mellitus tipo 2.

Diseño: Observacional con sistema de medición, descriptivo.

Institución: Departamento Académico de Ciencias Dinámicas, Facultad de Medicina, UNMSM.

Participantes: Pacientes con diabetes mellitus tipo 2.

Intervenciones: Se desarrolló encuestas socio- económicas a 300 pacientes con diabetes mellitus tipo 2, además de las medidas antropométricas; posterior a ello se tomó una muestra de sangre para los análisis bioquímicos.

Principales medidas de resultados: Peso, talla, IMC, índice cintura cadera (ICC), glucosa mg\%, triglicéridos $\mathrm{mg} \%$.

Resultados: De los 300 pacientes encuestados, 58,8\% correspondió al género masculino; 41,2\% tenía educación primaria, $44,1 \%$ educación secundaria y $14,7 \%$ educación superior. El $34 \%$ de la población presentó hiperglucemia, $58 \%$ obesidad de primer y segundo grado, $17,6 \%$ sobrepeso y $23,6 \%$ peso normal. El $64,7 \%$ de los pacientes no desarrollaba dieta alguna, $64,7 \%$ no tenía consejeria diabética, $59 \%$ no desarrollaba actividad física y presentó hiperglucemia. El $35 \%$ de los pacientes tenía control mensual de la enfermedad y sus niveles de glucosa estaban en los valores normales. Encontramos una correlación IMC / niveles de glucosa con la ayuda del coeficiente de Pearson $=0,524, p<0,01$.

Conclusiones: La relación IMC/ glicemia tuvo un coeficiente de Pearson $=0,524, p<0,01$, mostrando correlación moderadamente significativa para los indicadores.

Palabras clave: Diabetes mellitus, hiperglucemia, normoglicemia.

\section{Estudio del fenotipo de haptoglobinas en pacientes con diabetes mellitus tipo 1, en Lima, Perú}

Clever Arias, Miriam Inga, Raquel Oré, Luis Zapata, Carmen Aquije

Centro de Investigación de Bioquímica y Nutrición, Facultad de Medicina, UNMSM

Objetivos: Determinar el fenotipo de haptoglobina -1/1,1/2 y 2/2- predominante en una muestra de 36 pacientes con diabetes mellitus tipo I (DM tipo I).

Diseño: Observacional con sistema de medición, descriptivo.

Institución: Centro de Investigación de Bioquímica y Nutrición, Facultad de Medicina, UNMSM.

Participantes: Pacientes con diagnóstico de diabetes mellitus tipo I

Intervenciones: En 36 pacientes con diagnóstico de diabetes mellitus tipo I, se realizó medidas antropométricas, evaluaciones médicas en los pacientes diabéticos tipo II y, finalmente, se tomó una muestra de sangre para los análisis moleculares.

Principales medidas de resultados: Evaluación médica, electroforesis en agarosa.

Resultados: El 69,4\% de la muestra fue sexo femenino y 30,6 \% masculino; la edad promedio fue 28,3 $\pm 11,6$ y $26 \pm 11,6$ años para mujeres y hombres, respectivamente. La prevalencia del fenotipo de haptoglobina $1 / 1,1 / 2$ y $2 / 2$ fue $22,2 \%$, $38,9 \%$ y $38,9 \%$, respectivamente. Los pacientes del fenotipo $1 / 1$ presentaron menores complicaciones (retinopatías, cataratas, cardiopatías, peso bajo, neuropatías, glaucoma, dermatitis, infección urinaria), en comparación con los fenotipos $1 / 2$ y $2 / 2$. Las neuropatías afectaron por igual a pacientes con fenotipo $1 / 2$ y $2 / 2$.

Conclusiones: Empleando chi-cuadrado, no se encontró asociación estadísticamente significativa $(p>0,05)$ entre los fenotipos de Hp presente y las complicaciones asociadas a DM tipo I.

Palabras clave: Haptoglobina 1/1, 1/2 y 2/2, diabetes mellitus tipo 1, fenotipo. 